\title{
Real time portable genome sequencing for global food
}

\section{security [version 1; peer review: 1 approved, 1 approved with}

\section{reservations]}

\author{
Laura Boykin (D1), Ammar Ghalab1, Bruno Rossitto De Marchi (D1,2, Anders Savill1, \\ James M. Wainaina1, Tonny Kinene1, Stephen Lamb1, Myriam Rodrigues', \\ Monica Kehoe ${ }^{3}$, Joseph Ndunguru4 ${ }^{4}$ Fred Tairo ${ }^{4}$, Peter Sseruwagi 4 , \\ Charles Kayuki ${ }^{4}$, Deogratius Mark ${ }^{4}$ Joel Erasto ${ }^{4}$, Hilda Bachwenkizi ${ }^{4}$, Titus Alicai ${ }^{5}$, \\ Geoffrey Okao-Okuja5, Phillip Abridrabo (D5, Emmanuel Ogwok (D), \\ John Francis Osingada55, Jimmy Akono5, Elijah Ateka6, Brenda Muga6, \\ Samuel Kiarie 6
}

\footnotetext{
${ }^{1}$ School of Molecular Sciences, The University of Western Australia, Crawley, Western Australia, 6009, Australia

${ }^{2}$ School of Agriculture, Dept. of Plant Protection, São Paulo State University (UNESP), Botucatu (SP), CEP 18610-307, Brazil

${ }^{3}$ Crop Protection Branch, Department of Agriculture and Food Western Australia, South Perth, Western Australia, 6151, Australia

${ }^{4}$ Mikocheni Agricultural Research Institute (MARI), Dar es Salaam, Tanzania

${ }^{5}$ National Crops Resources Research Institute (NaCRRI), Kampala, Uganda

бomo Kenyatta University of Agriculture and Technology (JKUAT), Nairobi, Kenya
}

\section{V1 First published: $18 \mathrm{Jul} 2018, \mathbf{7 : 1 1 0 1}$ \\ https://doi.org/10.12688/f1000research.15507.1}

Latest published: $18 \mathrm{Jul}$ 2018, 7:1101

https://doi.org/10.12688/f1000research.15507.1

\section{Abstract}

Crop losses due to viral diseases and pests are major constraints on food security and income for millions of households in sub-Saharan Africa (SSA). Such losses can be reduced if plant diseases and pests are correctly diagnosed and identified early. Currently, accurate diagnosis for definitive identification of plant viruses and their vectors in SSA mostly relies on standard PCR and next generation sequencing technologies (NGS). However, it can take up to 6 months before results generated using these approaches are available. The long time taken to detect or identify viruses impedes quick, within-season decision-making necessary for early action, crop protection advice and disease control measures by farmers. This ultimately compounds the magnitude of crop losses and food shortages suffered by farmers. The MinION portable pocket DNA sequencer was used, to our knowledge globally for the first time, to sequence whole plant virus genomes. We used this technology to identify the begomoviruses causing the devastating cassava mosaic virus, which is ravaging smallholder farmers' crops in sub-Saharan Africa.

\section{Open Peer Review \\ Approval Status ? \\ 12 \\ version 1 \\ 18 Jul 2018

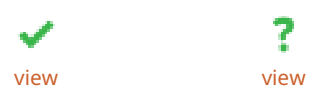 \\ 1. Ruth E Timme , U.S. Food and Drug \\ Administration, College Park, USA \\ 2. Alfonso Benítez-Páez (iD), Institute of Agrochemistry and Food Technology-Spanish National Research Council (IATA-CSIC), Valencia, Spain}

Any reports and responses or comments on the article can be found at the end of the article. 
Keywords

cassava, uganda, kenya, tanzania, nanopore, minion, SDG2

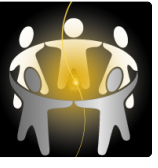

This article is included in the Nanopore Analysis

gateway.

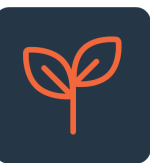

This article is included in the Agriculture, Food

and Nutrition gateway. 


\section{Corresponding author: Laura Boykin (laura.boykin@uwa.edu.au)}

Author roles: Boykin L: Conceptualization, Data Curation, Formal Analysis, Funding Acquisition, Investigation, Methodology, Project Administration, Resources, Software, Supervision, Validation, Visualization, Writing - Original Draft Preparation, Writing - Review \& Editing; Ghalab A: Data Curation, Formal Analysis, Investigation, Methodology, Writing - Original Draft Preparation, Writing - Review \& Editing; Rossitto De Marchi B: Data Curation, Formal Analysis, Investigation, Methodology, Software, Validation, Writing - Original Draft Preparation, Writing - Review \& Editing; Savill A: Conceptualization, Data Curation, Formal Analysis, Investigation, Methodology, Software, Supervision, Validation, Visualization, Writing - Review \& Editing; M. Wainaina J: Data Curation, Investigation, Methodology, Writing - Original Draft Preparation, Writing - Review \& Editing; Kinene T: Data Curation, Formal Analysis, Investigation, Methodology, Software, Validation, Visualization, Writing - Original Draft Preparation, Writing - Review \& Editing; Lamb S: Data Curation, Formal Analysis, Investigation, Methodology, Software, Visualization, Writing - Review \& Editing; Rodrigues M: Data Curation, Formal Analysis, Investigation, Methodology, Writing - Original Draft Preparation; Kehoe M: Conceptualization, Formal Analysis, Investigation, Methodology, Project Administration, Resources, Software, Supervision, Validation, Visualization, Writing - Original Draft Preparation, Writing - Review \& Editing; Ndunguru J: Conceptualization, Formal Analysis, Funding Acquisition, Investigation, Methodology, Project Administration, Resources, Supervision, Writing - Original Draft Preparation, Writing - Review \& Editing; Tairo F: Conceptualization, Project Administration, Resources, Software, Supervision, Validation, Writing - Original Draft Preparation, Writing - Review \& Editing; Sseruwagi P: Conceptualization, Data Curation, Formal Analysis, Investigation, Methodology, Project Administration, Resources, Software, Supervision, Validation, Visualization, Writing - Original Draft Preparation, Writing - Review \& Editing; Kayuki C: Data Curation, Formal Analysis, Investigation, Methodology, Project Administration, Resources, Software, Supervision, Validation, Visualization, Writing - Original Draft Preparation, Writing - Review \& Editing; Mark D: Data Curation, Formal Analysis, Investigation, Methodology, Software, Validation, Visualization, Writing - Original Draft Preparation, Writing - Review \& Editing; Erasto J: Data Curation, Formal Analysis, Investigation, Methodology, Software, Validation, Visualization, Writing - Original Draft Preparation; Bachwenkizi H: Formal Analysis, Investigation, Methodology, Software, Validation, Visualization, Writing - Original Draft Preparation; Alicai T: Conceptualization, Data Curation, Formal Analysis, Funding Acquisition, Investigation, Methodology, Project Administration, Resources, Software, Supervision, Validation, Visualization, Writing - Original Draft Preparation, Writing - Review \& Editing; Okao-Okuja G: Data Curation, Formal Analysis, Investigation, Methodology, Project Administration, Software, Supervision, Validation, Visualization, Writing - Original Draft Preparation; Abridrabo P: Data Curation, Formal Analysis, Investigation, Methodology, Software, Validation, Visualization, Writing - Original Draft Preparation; Ogwok E: Data Curation, Formal Analysis, Investigation, Methodology, Software, Validation, Visualization, Writing - Review \& Editing; Francis Osingada J: Data Curation, Formal Analysis, Investigation, Methodology, Software, Validation, Visualization, Writing Original Draft Preparation; Akono J: Formal Analysis, Investigation, Methodology, Software, Validation, Visualization, Writing - Original Draft Preparation; Ateka E: Conceptualization, Data Curation, Formal Analysis, Funding Acquisition, Investigation, Methodology, Project Administration, Resources, Software, Supervision, Validation, Visualization, Writing - Original Draft Preparation, Writing - Review \& Editing; Muga B: Data Curation, Formal Analysis, Investigation, Methodology, Software, Supervision, Validation, Visualization, Writing Original Draft Preparation, Writing - Review \& Editing; Kiarie S: Formal Analysis, Investigation, Methodology, Validation, Visualization, Writing - Original Draft Preparation

Competing interests: No competing interests were disclosed.

Grant information: This work was part-funded by the Crawford Fund, Australia (grant number WA-803-2017). The funders had no role in study design, data collection and analysis, decision to publish, or preparation of the manuscript.

Copyright: @ 2018 Boykin L et al. This is an open access article distributed under the terms of the Creative Commons Attribution License, which permits unrestricted use, distribution, and reproduction in any medium, provided the original work is properly cited.

How to cite this article: Boykin L, Ghalab A, Rossitto De Marchi B et al. Real time portable genome sequencing for global food security [version 1; peer review: 1 approved, 1 approved with reservations] F1000Research 2018, 7:1101 https://doi.org/10.12688/f1000research.15507.1

First published: 18 Jul 2018, 7:1101 https://doi.org/10.12688/f1000research.15507.1 


\section{Introduction}

The United Nations has listed Zero Hunger as one of the 17 global sustainable development goals to end extreme poverty by 2030. Plant viruses are a major constraint to crop production globally, causing an estimated $\$ 30$ billion in damage ${ }^{1}$ and leaving millions of people food-insecure ${ }^{2}$. In Africa, agriculture employs up to $50 \%$ of the workforce, yet only contributes $15 \%$ to the GDP on average ${ }^{3}$, suggesting that there is low productivity and limited value addition. This can be addressed through continued innovation in the fields of science and technology, as suggested in the Science Agenda for Agriculture in Africa (S3A) ${ }^{4}$. Sustainable management of plant viruses and their associated vectors must include efficient diagnostics for surveillance, detection and identification to inform disease management, including the development and strategic deployment of virus resistant varieties. To date, researchers have been utilizing conventional methods such as; PCR, qPCR, high-throughput sequencing (RNA-Seq, DNA-Seq) and Sanger sequencing for pathogen identification. However, these methods are both costly and time consuming, delaying timely control actions. The emergence of new tools for real-time diagnostics, such as the Oxford Nanopore MinION, have recently proven useful for the early detection of Ebola ${ }^{5}$ and $\mathrm{Zika}^{6,7}$, even in poorly resourced laboratories. For the first time globally, the MinION portable pocket DNA sequencer was used to sequence whole plant virus genomes. We used this technology to identify the begomoviruses causing the devastating cassava mosaic virus, which is ravaging smallholder farmers' crops in sub-Saharan Africa. Cassava, a carbohydrate crop from which tapioca originates, is a major source of calories for over 800 million people worldwide. With this technology, farmers struggling with diseased crops can take immediate, restorative action to improve their livelihoods based on information about the health of their plants, generated using a portable, real-time DNA sequencing device.

Portable DNA sequencing technology has great potential to reduce the risk of community crop failure and help improve livelihoods of millions of people, especially in low resourced communities. Plant diseases are a major cause of low crop productivity and viruses such as tobacco mosaic virus, tomato mosaic, tomato spotted wilt, potato leaf roll, Potato virus $\mathrm{X}$ and $\mathrm{Y}$ in potato, papaya mosaic, citrus tristeza, chilli leaf curl, and banana bunchy top have been implicated. In particular, cassava viruses are among the world's greatest risk to food insecurity. Losses caused by cassava mosaic disease (CMD) and cassava brown streak disease are estimated at US \$2-3 billion annually ${ }^{8}$.

\section{Results and discussion}

We visited smallholder farmers in Tanzania, Uganda and Kenya (Table 1) who are suffering yield shortages due to cassava virus infections. We utilized the MinION to test infected material and farmers were informed within 48 hours of the specific strain of the virus that was infecting their cassava, and a resistant cassava variety was deployed. The advantages of adopting this technology far outweigh the challenges (Table 2). Cassava mosaic begomoviruses were in high enough concentration that reads of whole genomes were obtained without an enrichment step (Table 1). As expected, the viral reads increased with the severity of the symptoms observed (Table 1). We detected a dual infection for a leaf sample with the severity score of 5 in Uganda. In addition, one asymptomatic plant in Tanzania had one viral read detected. The shortest time to obtain a viral read was $15 \mathrm{~s}$ (severity score 5) and the longest was $4 \mathrm{~h} 11 \mathrm{~m} 15 \mathrm{~s}$ (severity score 1).

MinION sequencing is superior to traditional methods of PCR identification, given its generation of whole genome sequences which enable the identification of the plant virus strain even if it becomes mutated or divergent, as it is not biased using primers that rely on known virus sequences. With regards to cassava, there are three major advantages of this technology. Firstly, improved diagnostics are required and real-time wholegenome sequencing will help develop diagnostic primers that are up-to-date. Secondly, this technology will assist with the development of resistant cassava varieties and will allow breeders to immediately test the varieties they are developing against different viral strains. Lastly, it ensures the delivery of the correct healthy uninfected planting material to farmers. In addition, we could detect virus in a plant before it showed symptoms (Table 1). Utilizing traditional PCR methods, three samples collected from farmer 1's field in Tanzania tested positive for EACMVs and none were positive for ACMV. The asymptomatic sample from Mikocheni Agricultural Research Institute (MARI) tested negative for both ACMV and EACMVs. There were eight fresh cassava leaf samples from Uganda found to be dually infected with ACMV and EACMV-UG using conventional PCR primers for ACMV and EACMV-UG. The primers used in this PCR yield products of $1000 \mathrm{bp}$ and 1500 bp for ACMV and EACMV-UG, respectively. In total, 12 Kenyan samples were tested and all but two (barcode 2 and 3) were found to be positive using conventional PCR (Table 1). Further studies are needed to verify our results regarding the sensitivity of the protocol for early detection of CMD in cassava, but these results are very promising for ensuring farmers receive clean planting material through the early detection of viral infection.

Nanopore sequencing technology has wide applications globally, but in East Africa these include: (a) crop improvement by screening for virus resistant germplasm and genetic diversity during breeding; (b) indexing of cassava planting materials for virus presence or absence to ensure that only clean materials in multiplication fields are distributed to farmers; (c) detection and identification of alternative plant species for cassava-infecting begomoviruses, so that farmers are advised to remove and/or grow crops away from such plants as a management strategy; and (d) virus and biodiversity studies.

\section{Methods}

Sample collection and DNA extraction

In Tanzania, three cassava mosaic disease (CMD) symptomatic cassava leaf samples (Figure 1, Table 1) were collected from the smallholder cassava farmer 1's field in Bagamoyo. Disease severity was assessed as described by Legg et $a .^{2}{ }^{2}$, where 1 is healthy and 5 shows severe symptoms of the disease, including leaf distortion and stunting of the plant. One more 


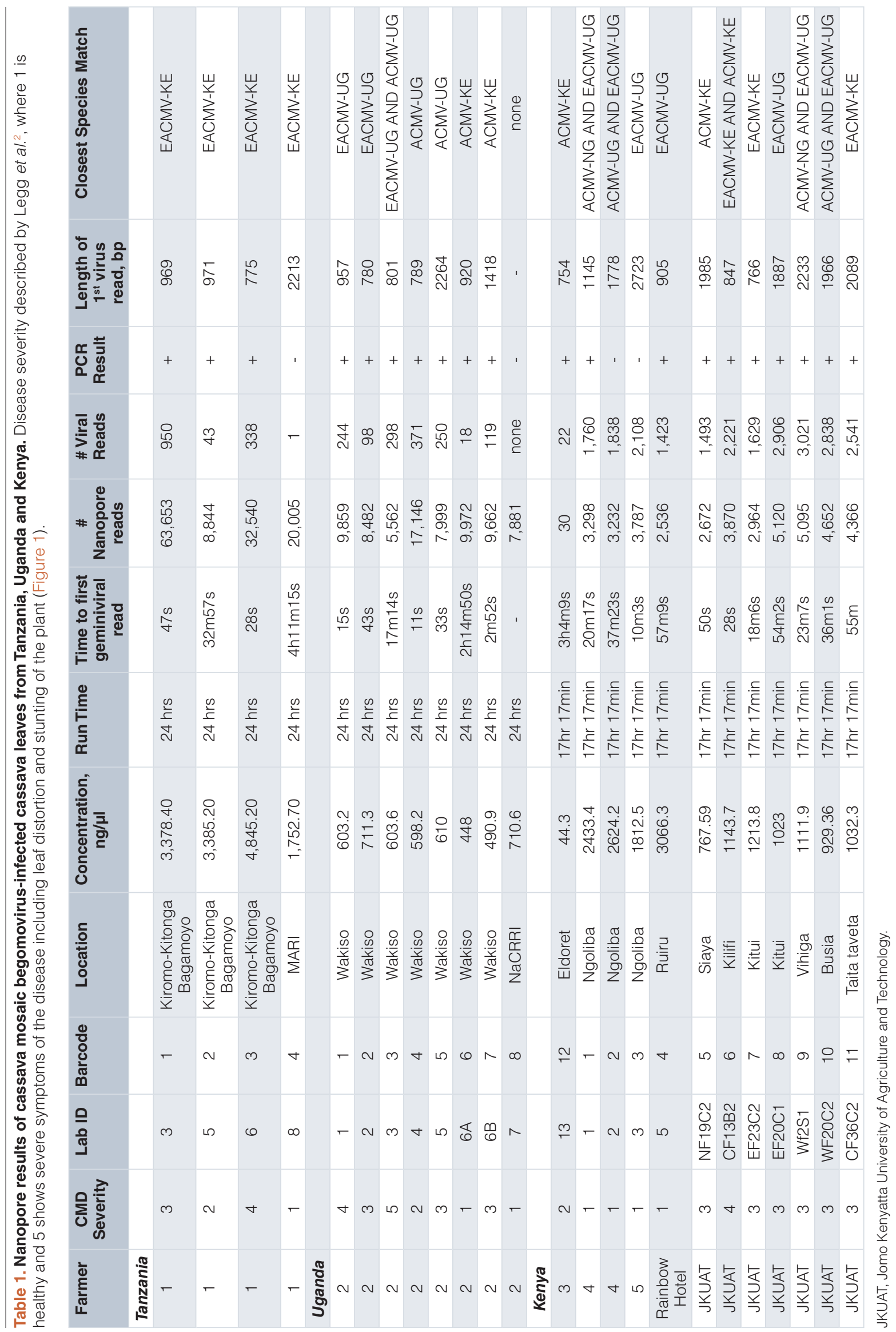


Table 2. Advantages and challenges of using the MinION portable DNA sequencer in Tanzania, Uganda and Kenya.

\begin{tabular}{|l|l|}
\hline Advantages & Challenges \\
\hline 1. Rapid - obtain results timely to support quick decision making on disease management & 1. Power \\
\hline 2. Expenses on sending samples for sequencing abroad removed & 2. Internet \\
\hline 3. High resolution of results and reliability & 3. Cost \\
\hline 4. Detection of mixed infections & 4. Computer access \\
\hline 5. Detection of virus in latently infected plants & 5. Shipping \\
\hline 6. Discovery of unknown viruses & \\
\hline 7. Minimising sample degradation and loss during shipping & \\
\hline 8. Virus-indexing for safe movement of germplasm & \\
\hline 9. Better plant health regulation, inspection/phytosanitary - improved international trade & \\
\hline
\end{tabular}

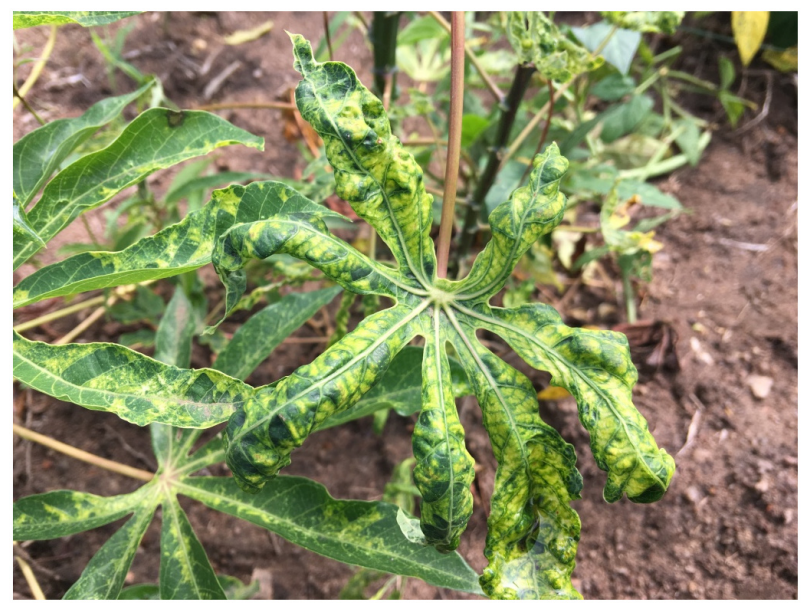

Figure 1. Cassava plant infected with cassava mosaic begomoviruses.

asymptomatic leaf sample was collected at MARI, Dar es Salaam. In total, seven CMD symptomatic plants were collected from farmer 2's farm in Wakiso district in Uganda. Both Tanzanian and Ugandan samples were collected in September 2017. A total of 12 samples from Kenya were collected in February 2018 from various sources (Table 1). High-quality DNA was isolated using the cetyl trimethylammonium bromide method ${ }^{9}$. Each DNA sample (Table 1) was quantified and the purity checked using a NanoDrop 2000c UV-vis Spectrophotometer (Thermo Fisher Scientific, Wilmington, DE, USA) was used to check the purity and quantity of DNA for each sample, and results were recorded in Table 1.

\section{Nanopore library preparation and sequencing}

In Tanzania and Uganda, the Rapid Barcoding kit SQK-RBK001 and MinION 9.4.1 flow cells (Oxford Nanopore) were used to process genomic DNA extracted using a standard CTAB method ${ }^{9}$. We utilized the Rapid Barcoding kit SQK-RBK004 with 9.4.1 flow cells in Kenya. DNA was diluted to $700 \mathrm{ng}$ as specified in the library protocol. The SQK-RBK001 (Sept 2017) and/or the SQK-RBK004 (Feb 2018) protocols were performed as described by the manufacturer. In Tanzania and Uganda, the MinION was run for $24 \mathrm{~h}$ instead of the recommended 48 $\mathrm{h}$, and in Kenya we had a total run time of approximately $17 \mathrm{~h}$ due to power interruptions.

\section{Nanopore bioinformatics}

In Tanzania and Uganda, Albacore 2.0.2 was used for base calling. In Kenya, Albacore 2.1.10 was used and the scripts were modified to reflect the newest rapid barcoding kit RBK004. Fastq files were imported into Geneious ${ }^{10}$ and a local blast database of all known cassava mosaic begomovirus whole genomes were downloaded from GenBank (program set to megablast, maximum hits $=1$, scoring (match mismatch 1-2, max e-value 1e-1, word size 28, gap cost=linear) and a local blast was performed on each of the reads generated using the Nanopore device. There are other bioinformatics pipelines that are used for Nanopore data, but the focus of our study was to use a simple, efficient analyses pipeline, which was a local blast database and a well-curated reference dataset ${ }^{11}$. To ensure we did not miss other begomoviruses or pathogens, we confirmed these in-field results by performing a post diagnostic blast of reads on the Nimbus Cloud at the Pawsey Supercomputing Center (Kensington, Australia) with blast 2.2.31 against the full NCBI nucleotide database to confirm results. For reference, the specific database used was $\{\$$ blastcmd $-\mathrm{db} n t / n t$ -info \} Database: Nucleotide collection (nt) 46,853,753 sequences; 170,830,796,758 total bases Date: Feb 20, 2018 5:04 PM. The data were processed into a blast archive using a blast script with the following parameters (Script attached) \{\$blastn -query "\$file" -db /mnt/nucdb/nt/nt -outfmt 11 -culling_limit 10 -out "out.\$file.asn" -num_threads 17$\}$ then converted into XML (for loading into Geneious) and HTML for viewing where possible.

\section{Verification of DNA sequencing results}

Traditional PCR was used to verify our DNA sequencing results. In Tanzania and Kenya, two primer pairs: EAB 555F/ EAB $555 \mathrm{~F}^{12}$ and JSP001/JSP002 ${ }^{13}$, which amplify $556 \mathrm{bp}$ and 774 bp, respectively, were used to detect East African CMVs 
(EACMVs) and African CMVs (ACMVs), respectively. Specifically, high quality DNA were isolated using CTAB method as described by Lodhi et al. ${ }^{9}$. Electrophoresis and spectrophotometric measurements were used to check the quality and quantity of DNA. PCR reactions were carried out in a $25 \mu \mathrm{l}$ volume, with $12.5 \mu \mathrm{l}$ (1X final concentration) of One Taq PCR 2X Master Mix (New England Biolabs), One Taq DNA Polymerase in an optimized buffer with $1.5 \mathrm{mM} \mathrm{Mg} 2^{+}$and $0.2 \mathrm{mM}$ each dNTPs in $1 \mathrm{X}$ final concentration. A total of $0.2 \mu \mathrm{M}(0.5 \mu \mathrm{l}$ of each) of primer sets were used in the reaction and nuclease-free water were added to $25 \mu \mathrm{l}$ final reaction volume. The template used was less than $500 \mathrm{ng}$ per reaction. PCR was carried out in 2720 Thermal Cycler (Applied Biosystems) with the following programme: 1 cycle of $3 \mathrm{~min}$ at $94^{\circ} \mathrm{C}$, then 30 cycles at $94^{\circ} \mathrm{C}$ for $45 \mathrm{~s}, 56^{\circ} \mathrm{C}$ for $45 \mathrm{~s}, 72^{\circ} \mathrm{C}$ for $1 \mathrm{~min}$, and a final cycle at $72^{\circ} \mathrm{C}$ for $7 \mathrm{~min}$. Electrophoresis of the PCR product was run on $1 \%$ agarose gel stained in ethidium bromide in $1 \mathrm{X}$ TAE buffer in a submarine gel electrophoresis unit and visualized using a BioDoc-It 210 Imaging System m-20V Transilluminator (Thomas Scientific).

In Uganda, the presence of ACMV and EACMV in each sample was detected using a pair of specific primers for ACMV, ACMV$\mathrm{AL} 1 / \mathrm{F}$ and ACMV-ARO/R, and primers specific for EACMV$\mathrm{UG} 2, \mathrm{UV}-\mathrm{AL} 1 / \mathrm{F}$ and ACMV-CP/R $3^{14}$. Specifically, the presence of ACMV and EACMV in each sample was detected using a pair of specific primers for ACMV, ACMV-AL1/F (5'-GCC GGA ATC CCT AAC ATT ATC -3') and ACMV-ARO/R (5'-GCT CGT ATG TAT CCT CTA AGG CCT G -3') and specific for EACMVUG2, UV-AL1/F (5'-TGT CTT CTG GGA CTT GTG TG -3') and ACMV-CP/R3 (5' - TGC CTC CTG ATG ATT ATA TGT C3') described by Zhou et al. ${ }^{14}$. The primers amplify about 1000 bp for ACMV and $1500 \mathrm{bp}$ for EACMV-UG2 of the Coat Protein and AV2 gene sequences of the ACMV and EACMV genomes, respectively. The PCR reaction was set up using GoTaq ${ }^{\circledR}$ Green Master Mix (Promega, Madison USA). Each of the $25 \mu \mathrm{l}$ PCR mix contained $12.0 \mu$ of $2 \mathrm{X}$ GoTaq ${ }^{\circledR}$ Green Master Mix [containing GoTaq ${ }^{\circledR}$ DNA polymerase, 2X Green GoTaq ${ }^{\circledR}$ Reaction Buffer (pH 8.5), $400 \mu \mathrm{M}$ dATP, $400 \mu \mathrm{M}$ dGTP, 400 $\mu \mathrm{M}$ dCTP, $400 \mu \mathrm{M}$ dTTP and $3 \mathrm{mM} \mathrm{MgCl}_{2}$ ], $1.0 \mu \mathrm{l}$ of forward primer, $1.0 \mu \mathrm{l}$ of reverse primer, $10.0 \mu \mathrm{l}$ of nuclease-free water (Amressco, Ohio, USA) and $1.0 \mu \mathrm{l}$ of DNA. PCR was performed using a Biometra professional thermocycler (Biometra, Gottingen, Germany) programmed as follows: $94{ }^{\circ} \mathrm{C}$ for $2 \mathrm{~min}$ for initial denaturation followed by 30 cycles of 94 ${ }^{\circ} \mathrm{C}$ for $1 \mathrm{~min}, 60{ }^{\circ} \mathrm{C}$ for $1.5 \mathrm{~min}, 72^{\circ} \mathrm{C}$ for $2 \mathrm{~min}$ and $72^{\circ} \mathrm{C}$ for $10 \mathrm{~min}$ for denaturation, annealing, extension and final extension, respectively. PCR amplicons were separated by electrophoresis in a $1 \times$ Tris-acetate-EDTA (TAE) buffer in a $1.2 \%$ agarose gel, stained with ethidium bromide $(0.1 \mathrm{mg} / \mathrm{ml})$ and visualized using a U:Genius3 (Syngene, Cambridge, UK) gel documentation system.

The image in Figure 1 was captured in Kiromo-Kitonga Bagamoyo, Tanzania by J.N. using a Samsung s8 smartphone.

An earlier version of this article can be found on bioRxiv (DOI: https://doi.org/10.1101/314526).

\section{Data availability}

Raw data for this study are available on figshare: DOI: https://doi.org/10.6084/m9.figshare.6667409 ${ }^{11}$.

\section{Competing interests}

No competing interests were disclosed.

\section{Grant information}

This work was part-funded by the Crawford Fund, Australia (grant number WA-803-2017).

The funders had no role in study design, data collection and analysis, decision to publish, or preparation of the manuscript.

\section{Acknowledgements}

Funding for the Kenyan trip was provided by the Crawford Fund. We also thank the participants from the University of Eldoret who assisted in the preparation of libraries for the Kenyan samples.
1. Subramanya Sastry K, Zitter TA: Management of Virus and Viroid Diseases of Crops in the Tropics. In Plant Virus and Viroid Diseases in the Tropics Vol. 2 Epidemiology and Management. 2014; 149-480. Publisher Full Text

2. Legg JP, Thresh JM: Cassava mosaic virus disease in East Africa: a dynamic disease in a changing environment. Virus Res. 2000; 71(1-2): 135-149. PubMed Abstract | Publisher Full Text

3. AFDB: African Development Bank. Annual Report. 2016. Reference Source

4. FARA: Science agenda for agriculture in Africa (S3A): "Connecting Science" to transform agriculture in Africa. Forum for Agricultural Research in Africa (FARA), Accra, Ghana. 2014. Reference Source

5. Quick J, Loman NJ, Duraffour S, et al.: Real-time, portable genome sequencing for Ebola surveillance. Nature. 2016; 530(7589): 228-232. PubMed Abstract | Publisher Full Text | Free Full Text

6. Faria NR, Sabino EC, Nunes MR, et al:: Mobile real-time surveillance of Zika virus in Brazil. Genome Med. 2016; 8(1): 97. PubMed Abstract | Publisher Full Text | Free Full Text

7. Quick J, Grubaugh ND, Pullan ST, et al: Multiplex PCR method for MinION and Illumina sequencing of Zika and other virus genomes directly from clinical samples. Nat Protoc. 2017; 12(6): 1261-1276.

PubMed Abstract | Publisher Full Text | Free Full Text

8. Scholthof KB, Adkins S, Czosnek H, et al:: Top 10 plant viruses in molecular plant pathology. Mol Plant Pathol. 2011; 12(9): 938-954. PubMed Abstract | Publisher Full Text

9. Lodhi MA, Ye GN, Weeden NF, et al:: A simple and efficient method for DNA extraction from grapevine cultivars and Vitis species. Plant Mol Biol Rep. 1994; 
12(1): 6-13

Publisher Full Text

10. Kearse M, Moir R, Wilson A, et al: Geneious Basic: an integrated and extendable desktop software platform for the organization and analysis of sequence data. Bioinformatics. 2012; 28(12): 1647-1649.

PubMed Abstract | Publisher Full Text | Free Full Text

11. Boykin L: Nanopore sequencing of cassava from Tanzania, Uganda and Kenya figshare. Fileset. 2018.

Data Source

12. Fondong VN, Pita JS, Rey ME, et al.: Evidence of synergism between African cassava mosaic virus and a new double-recombinant geminivirus infecting cassava in Cameroon. J Gen Virol. 2000; 81(Pt 1): 287-297. PubMed Abstract | Publisher Full Text

13. Pita JS, Fondong VN, Sangaré A, et al:: Genomic and biological diversity of the African cassava geminiviruses. Euphytica. 2001; 120: 115-125. Publisher Full Text

14. Zhou X, Liu Y, Calvert L, et al:: Evidence that DNA-A of a geminivirus associated with severe cassava mosaic disease in Uganda has arisen by interspecific recombination. J Gen Virol. 1997; 78(Pt 8): 2101-2111.

PubMed Abstract | Publisher Full Text 


\title{
Open Peer Review
}

\section{Current Peer Review Status:}

\section{Version 1}

Reviewer Report 24 August 2018

https://doi.org/10.5256/f1000research.16909.r36979

(C) 2018 Benítez-Páez A. This is an open access peer review report distributed under the terms of the Creative Commons Attribution License, which permits unrestricted use, distribution, and reproduction in any medium, provided the original work is properly cited.

\begin{abstract}
Alfonso Benítez-Páez
Microbial Ecology, Nutrition \& Health Research Unit, Institute of Agrochemistry and Food Technology-Spanish National Research Council (IATA-CSIC), Valencia, Spain

\section{Summary of the article}

Boykin et al present a pilot study aiming the application of real-time DNA sequencing for the detection of ACMV and EACMV in cassava plants in multiple crops of African East countries. This study represents the successful approaching of the most valuable feature of the MinION nanopore sequencing platform, its portability. At the same time, the authors made the maximum use of the singularities of the system by the translational application of their results, thus preventing the spread of plant viruses and to improve the crop efficiency by timely advising of farmers. This last exercise really highlights the value of such technology, particularly in the epidemiological surveillance and control of pathogens.
\end{abstract}

Notwithstanding, I have some minor concerns that if addressed they would constitute an added value to the approach described.

1) I strongly recommend that authors store and make publicly available the genetic information retrieved from different sequencing runs to a specialized repository such as ENA or GenBank.

2) It would be very informative for future studies in this field that Table 1 contains additional information the average or median values of the sequence identity derived from the comparison between nanopore reads and reference sequences. In a similar way, they should declare the level of relationship between ACMV and EACMV, in terms of genome-wide nucleotide identity, in order to disclose any potential misidentification given the high error rate of nanopore-derived DNA reads.

3) The authors must be aware that there is a strong effect derived from sequencing kits used, different from Uganda/Tanzania and Kenya. They should make some correlations between the CMD severity scoring and DNA reads retrieved independently accordingly to the kits used. 
4) In the same line of thoughts than above, it would be very elegant that authors will estimate the maximum time for expecting a viral DNA read just for setting a threshold and optimize the sequencing time. I have noticed that for $C M D$ severity $=1$, it took maximum $4 \mathrm{~h}$ to retrieve viral DNA reads, using the sequencing kit SQK-RBK001. Another different history was the utilization of SQK-RBK004 in Kenya, where apparently there is not a correlation between CMD severity and viral DNA reads retrieved. In the last cases, the apparently not symptomatic plants were detected as positive in less than one hour. The setting of a time threshold for a proper detection (getting enough number of reads to estimate reliable identification) would be useful to speed up the farmers' advising and consequently the reduction of risks for the spread.

Competing Interests: No competing interests were disclosed.

Reviewer Expertise: My expertise areas are the microbial genomics, nanopore DNA sequencing technology, molecular biology, and the massive DNA data analysis.

\section{I confirm that I have read this submission and believe that I have an appropriate level of expertise to confirm that it is of an acceptable scientific standard, however I have significant reservations, as outlined above.}

Reader Comment 13 Nov 2018

Edwin karoney, Masinde Muliro University of Science and Technology, Kenya

Great work! Outstanding technology!

Competing Interests: No competing interests were disclosed.

Reviewer Report 26 July 2018

https://doi.org/10.5256/f1000research.16909.r36183

(C) 2018 Timme R. This is an open access peer review report distributed under the terms of the Creative Commons Attribution License, which permits unrestricted use, distribution, and reproduction in any medium, provided the original work is properly cited.

\section{Ruth E Timme}

Center for Food Safety and Applied Nutrition, U.S. Food and Drug Administration, College Park, MD, USA

Boykin and co-authors present results from a pilot study exploring the use of MinION technology to detect a plant viral pathogen in real-time. Their data shows a huge advantage of using this hand-held sequence technology over the standard PCR methods. The authors propose implementing a MinION quality-control step on the plants before they are distributed, so that CMD-infected plants can be removed from the supply chain before they reach local farmers. This report is short, but its impact appears to be far-reaching. Most of my comments are minor editorial suggestions, but overall the writing and readability is excellent. 


\section{Minor revisions:}

Public availability of the DNA sequence data. While the authors technically made the sequence files public by posting to FigShare, the standard repository for DNA sequence data is the INSDC (NCBI/EBI/DDBJ). I highly urge the authors to create a BioProject at NCBI or EBI that houses the raw and assembled sequences (fasta files) for this effort so that other researchers in this area can easily build off this important work.

\section{Editorial comments:}

\section{Results and Discussion}

"We utilized the MinION to test infected material and farmers were informed within 48 hours of the specific strain of the virus that was infecting their cassava, and a resistant cassava variety was deployed." Consider converting to two sentences. One about using the MinION and the second to cover the response. Were resistant cassava plants really deployed within 48 hrs? wow.

"MinION sequencing is superior to traditional methods of PCR identification, given its generation of whole genome sequences which enable the identification of the plant virus strain even if it becomes mutated or divergent, as it is not biased using primers that rely on known virus sequences." Consider a minor re-write: "In general MinION sequencing is superior to traditional PCR methods of identification because the virus can be detected even when the PCR primers don't work, and 2) entire viral genome sequence is generated enabling the identification of the specific viral strain, along with other molecular information, which allows for a much higher resolution of surveillance.

"In addition, we could detect virus in a plant before it showed symptoms (Table 1)." Change to present tense to match the rest of the paragraph?

"Utilizing traditional PCR methods, three samples collected from farmer 1's field in Tanzania tested positive for EACMVs and none were positive for ACMV." Define EACMV and ACMV before abbreviation.

\section{Methods:}

"In Tanzania, three cassava mosaic disease (CMD) symptomatic cassava leaf samples (Figure 1, Table 1) were collected from the smallholder cassava farmer 1's field in Bagamoyo." CMD already defined in Intro.

"In Tanzania and Kenya, two primer pairs: EAB 555F/EAB 555F12 and JSP001/JSP00213, which amplify 556 bp and 774 bp, respectively, were used to detect East African CMVs (EACMVs) and African CMVs (ACMVs), respectively." Use the abbreviations here after adding the full names to the Results.

Competing Interests: No competing interests were disclosed.

Reviewer Expertise: I'm a Research Microbiologist for the US Food and Drug Administration. My research focuses on the validation of phylogenetic tools for molecular surveillance. I also manage a genome surveillance network for foodborne pathogens called the GenomeTrakr, that collects 
and publishes WGS data from food and environmental isolates.

I confirm that I have read this submission and believe that I have an appropriate level of expertise to confirm that it is of an acceptable scientific standard.

The benefits of publishing with F1000Research:

- Your article is published within days, with no editorial bias

- You can publish traditional articles, null/negative results, case reports, data notes and more

- The peer review process is transparent and collaborative

- Your article is indexed in PubMed after passing peer review

- Dedicated customer support at every stage

For pre-submission enquiries, contact research@f1000.com 